\title{
REPRESENTING A DISTRIBUTION BY STOPPING A BROWNIAN MOTION: ROOT'S CONSTRUCTION
}

\author{
Shey Shiung Sheu
}

A closed subset $C$ of $[0, \infty] \times[-\infty, \infty]$ is called a barrier if

(i) $(\infty, x) \in C, \forall x$,

(ii) $(t, \pm \infty) \in C, \forall t$,

(iii) $(t, x) \in C$ implies $(s, x) \in C, \forall s \geqslant t$.

Given a Brownian motion $(B(t))$ starting at the origin and a barrier $C$, let $\tau(C)$ be $\inf \{t:(t, B(t)) \in C\}$. A random variable $X$ (or a distribution $F$ ) is called achievable if there exists a barrier $C$ so that $B(\tau(C))$ is distributed as $X(F)$. In this paper we shall show that if $X$ is bounded above or below with finite mean or if $X$ has zero mean and $E\left(|X| \log ^{+}|X|\right)<\infty$ then $X$ is achievable. This result gives a partial answer to a problem raised by Loynes [7].

\section{Introduction}

In dealing with various limit theorems for sums of independent random variables, skorohod (see [9], page 163) introduced a method to imbed a mean-zero random variable $X$ into a Brownian motion $B(t), t \geqslant 0$, starting at the origin; that is, he found a stopping time $\tau$ (relative to a filtration generally larger than the Brownian filtration) so that $B_{\tau}$ has the same distribution as $X$ (denoted by $B_{\tau} \sim X$ ) and, furthermore, $E\left(X^{2}\right)=E(\tau)$. If one requires $\tau$ to be a stopping time relative to the Brownian filtration ( $\tau$ depends only on Brownian paths), whether such $\tau$ can be still constructed has been a research problem for many authors

Received 28 January 1986.

Copyright clearance Centre, Inc, Serial-fee code: 000-9727/86 $\$ A 2.00+0.00$. 
(see Root [8], Dubins [6], Chacon and Walsh [4], Azéma and Yor [1], Bass [2], Vallois [10], etc.). Among these constructions, Root's seems most intuitive. His stopping time is the hitting time of a certain set in the compactified time-state space $H \equiv[0, \infty] \times[-\infty, \infty]$. A closed set $C$ in $H$ will be called a barpier if

(i) $(\infty, x) \in C$ for all $x \in C$,

(ii) $(t, \pm \infty) \in C$ for all $t$,

(iii) $(t, x) \in C$ implies $(s, x) \in C$ for all $s \geqslant t$.

The space of barriers will be compact under the Hausdorff metric. For a barrier $C$, let $\tau(C)=\inf \{t, B(T)) \in C\}$. Root proved that if $X$ has zero mean and finite variance, then there exists a barrier $C$ so that $B(\tau(C)) \sim X$, and $E(\tau(C))=E\left(X^{2}\right)$. Loynes [7] defines a random variable $X$ to be achievable if there exists a barrier $C$ so that $B(\tau(C)) \sim X$. He posed the problem of finding conditions for $X$ to be achievable. In this respect, any $X$ with zero mean and finite variance is achievable; any degenerate random variable is achievable and, therefore, being zero-mean is not a necessary condition. In fact, Loynes [7] showed that if $X$ is concentrated on a half line $(-\infty, b]$ and $E(X) \geqslant 0$, or on $[a, \infty)$ and $E(X) \leqslant 0$, then $X$ is achievable. He also pointed out that if $X$ is achievable, then $E\left(|X|^{p}\right)<\infty$ for all $p, 0<p<1$. Unfortunately, Loynes' results do not cover important cases such as Poisson distributions $(X$ concentrated on the positive half line but $E(X)>0$ ). In this paper, we shall improve his results.

\section{Main results}

Call a sequence of random variables $\left\{X_{n}\right\}$ stochastically bounded if $\forall \varepsilon>0, \exists A>0$ such that $P\left(\left|X_{n}\right| \geqslant A\right) \leqslant \varepsilon$ for all $n$.

LEMMA 2.1. Let $\left\{C_{n}\right\}_{n=1}^{\infty}$ be a sequence of barriers such that $C_{n} \rightarrow C_{\infty}$. Then the corresponding hitting times $\tau\left(C_{n}\right) \rightarrow \tau\left(C_{\infty}\right)$ in probability. In particular, if $C_{\infty}$ consists of points at $\infty$ only, then $\tau\left(C_{n}\right)$ is not stochastically bounded.

Proof. This is just a rephrase of Lemma 1 in Loynes [7]. 
LEMMA 2.2. Let $\left\{X_{n}\right\}_{n=1}^{\infty}$ be a sequence of random variables such that $X_{n}$ converges to $X_{\infty}$ in distribution. Let $\left\{C_{n}\right\}_{n=1}$ be a sequence of barriers such that $C_{n} \rightarrow C_{\infty}$ and $C_{\infty}$ consists of at least one finite point. If $B_{\tau\left(C_{n}\right)} \sim X_{n}, 1 \leqslant n<\infty$, then $B_{\tau\left(C_{\infty}\right)} \sim X_{\infty}$.

Proof. By Lemma 2.1, $\tau\left(C_{n}\right)+\tau\left(C_{\infty}\right)$ in probability. By assumption, $P\left(\tau\left(C_{\infty}\right)<\infty\right)=1$. Therefore, there exists a subsequence $\tau\left(C_{n}\right) \rightarrow \tau\left(C_{\infty}\right)$ almost surely. By the continuity of Brownian paths, we conclude $B_{\tau\left(C_{\infty}\right)} \sim X_{\infty}$

THEOREM 2.3. Any random variable $X$ bounded below or above with finite mean is achievable. In particular, the Poisson random variable is achievable.

Proof. Without loss of generality, we may assume that $X \geqslant b>-\infty$. By Loynes' results, we may also assume $M=E(X)>0$. Let

$$
Y_{n}= \begin{cases}k M & \text { with probability } \frac{1}{2}\left(1-\frac{1}{n}\right) \\ b & \text { with probability } \frac{1}{2}\left(1-\frac{1}{n}\right) \\ -\frac{1}{2}(n-1)(k M+b) & \text { with probability } \frac{1}{n},\end{cases}
$$

where $k$ is chosen so that $k>0,(k-2) M+b>0$. Since $Y_{n}$ has. mean zero and finite variance, it is achievable and the barrier can be expressed as $\left\{(t, x): t \geqslant 0, x=k M\right.$ or $\left.-\frac{1}{2}(n-1)(k M+b)\right\} \cup$ $\left\{(t, x): t \geqslant t_{n}, x=b\right\}$ for some $t_{n}>0$. Let $x_{n}=X$ if $X \leqslant n$; $X_{n}=n$ if $X>n$, and let $M_{n}=E\left(X_{n}\right)$. Let

$$
Z_{n}=\left\{\begin{array}{cl}
x_{n} & \text { with probability } 1-\frac{1}{n} \\
-(n-1) M_{n} & \text { with probability } \frac{1}{n}
\end{array}\right.
$$

$Z_{n}$ has mean zero and finite variance and hence, is achievable. The corresponding barrier can be expressed as $c_{n}=\{(t, x): b \leqslant x \leqslant n$, $\left.t \geqslant t_{n}(x)\right\} \cup\left\{(t, x): t \geqslant 0, x=-(n-1) M_{n}\right\}$. For $n \geqslant k M$, let $t_{n}^{\prime}=\inf \left\{t_{n}(x): b \leqslant x \leqslant k M\right\}$. Since $n>k M,-(n-1) M_{n}>-\frac{1}{2}(n-1)(k M+b)$, 
we have $t_{n}^{\prime} \leqslant t_{n}$. But $Y_{n}$ converges in distribution to $Y$, where $P(Y=k M)=P(Y=b)=\frac{1}{2}$. Therefore, $t_{n}$ will converge to a finite number and consequently, $C_{n}$ will not diverge to infinity. Since $z_{n}$ converges to $X$ in distribution, $X$ is achievable by Lemma 2.1 and Lemma 2.2 .

THEOREM 2.4. If $X$ is a random variable satisfying $E(X)=0$, $E\left(|X| \log ^{+}|X|\right)<\infty$, then $X$ is achievable.

Proof. We may assume that $X$ is neither bounded above nor bounded below. Then there exist sequences $a_{n} \rightarrow-\infty, b_{n} \rightarrow \infty$ such that if $X_{n}=X$, when $a_{n} \leqslant X \leqslant b_{n} ; X_{n}=0$, when $X<a_{n}$ or $X>b_{n}$, and $E\left(X_{n}\right)=0$. of course, $X_{n}$ is achievable. Let $\tau_{n}=\tau\left(C_{n}\right)$ be the stopping time such that $B_{\tau_{n}} \sim X_{n}$ and $E\left(\tau_{n}\right)=E\left(X_{n}^{2}\right)$. By the famous Burkholder-Gundy's inequality (see Theorem 6.1 in Burkholder [3]), we have

$$
E\left(\sqrt{\tau_{n}}\right) \leqslant c E\left(\sup _{0 \leqslant t \leqslant \tau_{n}}|B(t)|\right) .
$$

By Doob's inequality (see Doob [5], page 317) and the fact that $\sup _{0 \leqslant t \leqslant \tau_{n}}|B(t)|$ is bounded, we have

$$
\begin{aligned}
E\left(\sup _{0 \leqslant t \leqslant \tau}|B(t)|\right) & \leqslant \frac{e}{e-1}+\frac{e}{e-1} E\left(\left|X_{n}\right| \log ^{+}\left|x_{n}\right|\right) \\
& \leqslant \frac{e}{e-1}+\frac{e}{e-1} E\left(|X| \log ^{+}|X|\right) \\
& <\infty .
\end{aligned}
$$

Hence, $\left\{E\left(\sqrt{\tau_{n}}\right)\right\}$ is bounded, which implies $\left\{\tau_{n}\right\}$ is stochastically bounded. Since $X_{n}$ converges to $X$ in distribution, $X$ is achievable by Lemma 2.1 and Lemma 2.2 .

\section{References}

[1] J. Azéma and M. Yor, "Une solution simple au probléme de Skorokhod", Sem. Prob. XIII, Lecture Notes in Math. 721 (1979), Springer, 90-115. 
[2] R.F. Bass, "Skorohod imbedding via stochastic integrals", Sem. Prob. XVII, Lecture Notes in Math., 986 (1983), Springer, 221-224.

[3] D.L. Burkholder, "Distribution function inequalities for martingales", Ann. Prob., (1) 1 (1973), 19-42.

[4] R.V. Chacon and J.B. Walsh, "One-dimensional potential embedding", Sem. Prob. X, Lecture Notes in Math., 511 (1976), Springer, 19-23.

[5] J.L. Doob, Stochastic Process (Wiley, New York, 1953).

[6] L.E. Dubins, "On a theorem of skorohod", Ann. Math. Stat., 39 (1968), 2094-2097.

[7] R.M. Loynes, "Stopping times on Brownian motion: some properties of Root's construction", 2. Wahr. verw Gebiete, 16 (1970), $211-218$.

[8] D.H. Root, "The existence of certain stopping times on Brownian motion", Ann. Math. Stat., (2) 40 (1967), 715-718.

[9] A.V. Skorokhod, Studies in the Theory of Random Processes (AddisonWesley, Reading, 1965).

[10] P. Vallois, "Le probleme de skorokhod sur R: une approche avec le temps local", Sem. Prob. XVII, Lecture Notes in Math., 986 (1983), Springer, 227-239.

Institute of Applied Mathematics,

National Tsing Hua University,

Hsinchu, Taiwan 30043,

Republic of China. 